\title{
THE COMPARISON OF COMPETITIVE BALANCE BETWEEN SUPER RUGBY (SANZAR) AND ENGLISH PREMIERSHIP RUGBY: A CASE STUDY FROM 1996-2014 SEASON OR NOT ATTRACTIVE - NO PEOPLE - NO MONEY
}

\author{
Robert KUCHAR ${ }^{1}$ and Andy MARTIN ${ }^{2}$
}

\begin{abstract}
Most sports are attractive because they are almost unpredictable. The more the competitiveness of league teams, the harder to predict the games and as a result, that league will be more attractive. Message is: more attractive leagues $=$ bigger audience $=$ more attractive for sponsorship $=$ more money in sport. Competitive balance $(C B)$ refers to the balance in sport capabilities of teams. The aim of this paper was to compare the competitive balance between Super Rugby league named SANZAR, which consist of three nations (New Zealand, Australia and South Africa) and English Premiership Rugby League in 1996-2014 seasons and compare them. The data were secondary and collected from the final tables.

It was used five models in this study: the three-club and five-club concentration ratio (C3 and C5) and $C 3 / C 5$ index of competitive balance $(C 3 I C B / C 5 I C B)$ were used to analyse the data. The less the index $C 3 I C B / C 5 I C B$ and $C 3 / C 5$ are, the more competitive balance is, and conversely. Standard deviation of game results, the ratio of actual and ideal standard deviation wins, numbers of winners and a placement in the $k$-th place.
\end{abstract}

The results showed that the Salary cap in Premiership League does not work very effectively and the competitive balance is in last few seasons still worst and worst. Super Rugby has mirror position to Premiership. Last few years are the competitive balance on the right track. Comparison result is for Super Rugby strategy with involving more teams to the league.

\section{KEY WORDS \\ Competitive Balance, Sanzar, Super Rugby, Premiership Rugby, Salary Cap}

\section{INTRODUCTION}

In 1996 as SANZAR was formed, the Super 12 was created, a professional competition and the first official 'international domestic' tournament featuring the strongest teams - which would become franchises - across New Zealand, South Africa and Australia. Five franchises were created in New Zealand, merging the 26 domestic unions. The Kiwi sides are representative of their 'catchment areas' (the collection of unions that make up the franchise). The three Australian teams were the traditional rugby states of New South Wales and Queensland and a new team from the ACT called the Brumbies. For the first three years of the competition, South Africa determined its sides via qualification in the Currie Cup, with the four semi-finalists from the previous season taking part,

\footnotetext{
${ }^{1}$ Department of Strategy, Faculty of Business Administration, University of Economics, Prague, Czech Republic

${ }^{2}$ Department of School of Sport and Exercise, Massey University, Palmerstone North, New Zealand
} 
before they loosely followed the Kiwi model and created franchises from combined Currie Cup unions. In 2006 Super 14 came into fruition, with the Western Force and Cheetahs joining the fold, naturally extending the round-robin regular season competition, while the Super Rugby Final's Series format of the top four playing semi-finals continued. Effective in 2011, Super Rugby expanded to 15 teams - with the Melbourne Rebels the new franchise - and split into three conferences, each with five teams and based in one of the three nations. At the same time, the regular season expands to 16 matches ( 8 homes, 8 away). Each team will play a double round-robin within its home conference, and play single matches against four teams from each of the other conferences. ${ }^{3}$

The world's most competitive domestic rugby union competition is now Aviva Premiership Rugby.

The top flight league has evolved over time since beginning in 1987 when the Courage Leagues were formed - a league pyramid that had more than 1,000 clubs playing in 108 leagues - each with promotion and relegation. Previous to this, only friendlies were played and a regional cup competition existed. The official sponsors of the league had been changed couple time in the history. By the end of the 1990s the Premiership had become the Zurich Premiership and had developed into the most competitive club competition in the world. Over the years the format of the league developed and evolved, with the play-offs and a wildcard introduced. Aviva Premiership Rugby consists of 22 rounds with round 22 taking place on Saturday 16th May 2015. The club that tops the Aviva Premiership Rugby table after round 22 will automatically qualify for home advantage in the Aviva Premiership Rugby semi-final against the club that finishes fourth. The club that finishes second will qualify for home advantage in the other Aviva Premiership Rugby semifinal against the club that finishes third. The two Aviva Premiership Rugby semi-final matches take place over the weekend of $22 \mathrm{nd} / 23 \mathrm{rd} / 24$ th May 2015 and the winning clubs went forward to the prestigious Aviva Premiership Rugby Final at Twickenham on Saturday 30th May 2015. The club that wins the Aviva Premiership Rugby Final is being Aviva Premiership Rugby Champions. ${ }^{4}$

The advantages of professionalism in sport are very common - to start providing money for making sport was a stimulus for players and teams to maximize their success and to give athletes the best resources to perform at the very best of their ability. However, professionalism can increase the display, the impact of money, it can also undermine the competiveness of competitions. Several studies have shown that professional sports are under a huge weight by what Neale (1964) termed the 'peculiar economics of sport'. In particular, the problem of money torments the industry, to the point that it has become an appropriate factor. Clearly money and the wealth of people plays have an increasingly important role in sport. In the context of professional rugby, the wealthy have the ability to purchase international rugby clubs and to have the luxury of being able to afford the top international players. In competitions where there is instability of wealth across teams, money has the effect of reducing the distribution of player talent and effectively creating uneven teams (McMillan, 1997). Result is that, sporting competitions need some limitations on economic competition to create competitive balance as well as to incite sustainability.

The question of how the estimated demand for sporting events is influenced by the probability of the teams wins, sport economists have addressed from the outset of this scientific discipline. In the $50 \mathrm{~s}$ and $60 \mathrm{~s}$ of the 20th century came to the view that heavy dominance is killing interest and reduced demand. As an outcome was that, consumers demand uncertainty and the result of increasing demand uncertainty increases. This theory became the basis for many of the recommendations in this field.

\footnotetext{
${ }^{3}$ Official web side of SANZAR Rugby: (http://www.sanzarrugby.com/superrugby/about-super-rugby/)

${ }^{4}$ Official web side of Premiership Ruby: (www.premiershiprugby.com/history/)
} 
Competitive balance relates to the balance in sport and among sport teams (Michie et al., 2004). The uncertainty in sports results is analyzed through various methods; however Szymanski (2003) differentiates among three kinds of uncertainty:

a) Match uncertainty that relates to uncertainty about a result of a particular game (between two teams)

b) Season uncertainty that relates to uncertainty about results in the course of a season

c) Championship uncertainty that relates to dominance of limited number of teams in a league or several consecutive seasons

However, every attempt to attract attention and audience through competitive balance can be seen as inappropriate from a special team's or fans ${ }^{6}$ point of view, especially if the suspect that the probability of winning is high (Jennet, 1984). If the result of the game is predictable, the people will be more worried than if the game starts soon (Jennet,1984). That is why the league management should provide appropriate reasons to increase competitive balance (Konig, 2000). It is obvious that the competitions need a certain level of competitive balance to survive and to increase quality. Without competitive balance, the games are easily predictable and as a result they lose attractiveness. The lack of competitive balance means that the number of viewers (both at the stadium and in front of a TV) is not at its maximum (Michie at al., 2004). Quirk a Fort (1992) claim that one of the reasons why people love teams sports is excitement resulting from uncertainty of results of league competitions.

To attract fan's attention, all strength and weaknesses should be assessed so that the uncertainty of results is possible (Quirk et al, 1992). Competitive balance is important due to the fact that provided all the factors are considered the same, the uncertainty of the game result will attract people's attention and their interest to watch matches (on TV or at the stadium). If the league is not balanced, it is very hard to gain earnings from tickets or selling TV rights. The same applies to the relations with sponsor; if the league is not appealing to viewers, it is not attractive for sponsors either. That is the reason why $i$ tis very important to increase and keep competitive balance so that the demand after sports clubs, matches, league and sports product overall is maximized. That is the main reason why different sports (leagues) adopted regulation measures to redistribute revenues in order to influence the balance of the competition. Competitive balance leads to balanced league as well. On the contrary, unbalanced leagues can face various problems and threats such as bankruptcy, corruption, threats from competing leagues, etc. Stronger and richer teams advance to premier leagues where they earn more financial resources and become even richer. This way, the gap among the teams widens even further. Competitive balance is crucial for league attractiveness. (Michie et al., 2004)

\section{Match uncertainty}

Impact match result uncertainty in demand and interest in this battle is being studied mainly through two characteristics. These are the number of spectators at the stadium and the TV audience. The level of outturn uncertainty match can be measured in several ways. Currently, the most used prematch odds betting, which are generated in a competitive market and reflect the likelihood of match result uncertainty. But this is not completely smooth. One of the drawbacks is that the classes are created in a moment in time than that when viewers are buying tickets available. Empirical studies that examine this issue, however, the hypothesis of great interest and attendance at the great uncertainty in the results do not confirm much. On the contrary, many of them show that audiences want to see his primary victory of the home team, so number of spectators is higher if the home team is the favorite high price. So the theory of outturn uncertainty does not match up to the same weight as previously thought. More recently, however, it examines how a match uncertainty impact on the audience viewing transmissions on television. Viewers, who watch matches at home, are not 
as interested in the outcome of the match and prefer mainly viewing experience transfer. In this case, the results may be wrestling uncertainty analysis of the relationship and the TV audience different than it is in attendance.

\section{$\underline{\text { League uncertainty }}$}

Theory uncertainty league results described it, what is the probability that one team or group of teams have dominated the competition in a particular season or over several seasons. League is a long-term competition and so there is no need independent variable, such as odds betting, which defines the level of uncertainty league results. The key question is what should be the division victories for items between teams in order to maximize overall attendance of league. Does the controversy whether increasing uncertainty league results has resulted in an increase of the total viewing attendance? The issue was or it currently spends time many sports economists and empirical studies associating attendance at matches and won the teams, but do not always have identical results. Some studies even show that less balanced competition can achieve higher overall spectator attendance as balanced competition.

From the above information indicates that there is no clear answer as to whether the increasing uniformity and increasing insecurity is increasing interest and demand for sports competition.

\section{$\underline{\text { Salary Cap }}$}

The salary cap is defined as a form of wage maxima, which may be imposed on total payments to players of a club or the league as a whole (Dabscheck, 2004). "Effectively, a salary cap restricts how much teams can pay their players, ultimately preventing the inflation of salaries" (Staudohar, 1999: 3). The idea of salary caps was introduced by Rottenberg (1956) who anticipated salary caps "as a possibility, let teams bid for players and players accept offers, subject only to the constraint that a ceiling is imposed on the salaries that may be paid to individual players" (256-257). It was proposed that the salary cap allowed a team to assemble a more competitive roster paying less than the maximum. As mentioned above, the rising salaries are having huge consequences on the success of sports competitions and the salary cap is a way to limit its effect.

The salary cap is designed to ensure that all teams are spending equal amounts on player salaries, so that money cannot be used by wealthy teams to stock their teams with the all the best players. Ideally, if all teams are spending under the salary cap and have somewhat equal resources in terms of coaches and management, the competition should be fairly balanced. Furthermore, the appeal of the cap to owners is that it is a way to control salary costs. Vrooman (2000) also suggests salary caps act as a binding rule and restrict the mobility of professional players as well.

In conclusion, competitive balance has been investigated in world leagues and in various years. The present study attempts to investigate the competitive balance between the Super Rugby league and English Premiership League from 1996-2014 and compare them.

\section{MATERIALS AND METHODOLOGY}

This study is descriptive and the data are secondary and were collected from valid documents and league tables. This study aims to investigate competitive balance between to leagues. Premiership with salary cap regulation and Super Rugby, with no regulation in 1996-2014 seasons and compare them. Five models were used in this study: 
1. The Three and Five-Club Concentration Ratio, also we can call it real values (C3 and C5) and the $\mathrm{C} 3$ and $\mathrm{C} 5$ Index of Competitive Balance, also we can call it empirical values (C3ICB, C5ICB)

2. Standard deviation of game results

3. Relative standard deviation (RSD)- The ratio of actual and ideal standard deviation wins

4. Number of winners

5. A placement in the $\mathrm{k}$-th place

The above models were used in many studies (Dejonghe, 2006; Novotny, 2006; Szymanski, 2003; Feddersen, 2006; Tenreiro, 2006; Brunelli, 2006; Gouget et al., 2006; Michie et al., 2004). As these models were economic and mathematic indexes, it was not necessary to approve their validity and reliability.

1. In a totally balance league, C3ICB and C5ICB always equals 100 (Michie et al., 2004). An obtained $\mathrm{C} 3 \mathrm{ICB}$ and $\mathrm{C} 5 \mathrm{ICB}>100$ shows a lower balance in that league. Index is an indicator of the static competitive equilibrium. C3 and C5 ("ideal values") in a totally balanced league changes by a change in the number of teams and as the leagues of this study consist of 12,14 and 15 teams:

$\mathrm{C} 3=0.25$ in a 12 -team league $\mathrm{C} 3=0.214$ in a 14 -team league $\quad \mathrm{C} 3=0.2$ in a 15 -team league

$\mathrm{C} 5=0.416$ in a 12 -team league $\quad \mathrm{C} 5=0.357$ in a 14 -team league $\quad \mathrm{C} 5=0.333$ in a 15 -team league

$\mathrm{C} 3 / \mathrm{C} 5$ ratio= total points won by the top five clubs/total number of points won by all clubs

$\mathrm{C} 3 / \mathrm{C} 5$ Index of Competitive Balance $=\mathrm{C} 3$ or $\mathrm{C} 5$ ratio $/(3$ or $5 / \mathrm{N}) * 100$

or, is it possible to say

Empirical value $(\mathrm{CICB})=($ ideal value/real value $) * 100$

Where " $\mathrm{N}$ " is the number of teams in the league.

2. Standard deviation of game results show us how to win in the league diverted from the league average. In the case of competition where matches cannot finish in a draw the league average of 0.5 .

3. RSD - the ratio of actual and ideal standard deviation extends the previous indicator of relationship to the situation which would occur in ideal distribution victories. It allows us to compare the actual deviations from the ideal state. Standard deviation in the ideal state in the tender without draws calculated as: $\sigma \mathrm{i}=0.5$ / $\mathrm{N}$, where $\mathrm{N}$ is the number of matches that each team plays.

4. Number of winners, respectively frequency of champions examines how often changing over time teams to master position. This method presents and is regularly substituted and even distribution of champions or a small group of teams, respectively one team in the competition dominates. It is an easily measurable indicator of clear meaningful.

5. A placement in the k-th positions is the latest way of measuring the competitive equilibrium, which is used in this paper. We are interested not only who is at the first place, but how many teams are placed in positions in the second, third, fourth, respectively k-th positions. It allows us to determine whether these positions receive wide number of teams or they are just points of the domain of a small group of clubs. What to do $\mathrm{k}$-th place finish in the period more teams, the competition is more balanced.

\section{RESULTS}


Table 1 and charts shows C3/C5 (ideal value), C3/C5 ratio (real value) and C3/C5 Index of Competitive Balance (C3ICB /C5ICB) for the selected seasons. 
Table 1: C3ICB/C5ICB, C3/C5 ratio, C3/C5 - ideal value in selected seasons

\begin{tabular}{|c|c|c|c|c|c|c|c|c|c|c|c|c|c|}
\hline \multicolumn{7}{|c|}{ Super Rugby League } & \multicolumn{5}{|c|}{ Premiership League } & \multirow[b]{2}{*}{$\begin{array}{c}\text { C3 } \\
\text { (IV } \\
\text { ) }\end{array}$} & \multirow[b]{2}{*}{$\begin{array}{c}\text { C5 } \\
\text { (IV } \\
\text { ) }\end{array}$} \\
\hline $\begin{array}{c}\text { Yea } \\
\text { r* }^{*}\end{array}$ & $\begin{array}{c}\text { C3I } \\
\text { CB } \\
\text { (E.V } \\
.)\end{array}$ & $\begin{array}{c}\text { C5I } \\
\text { CB } \\
(\mathrm{E} . \mathrm{V} \\
.)\end{array}$ & $\begin{array}{c}\text { C3 } \\
\text { ratio } \\
\text { (R.V.) }\end{array}$ & $\begin{array}{c}\text { C5 } \\
\text { ratio } \\
\text { (R.V.) }\end{array}$ & $\begin{array}{l}\text { C3 } \\
\text { (I. } \\
\text { V.) }\end{array}$ & $\begin{array}{c}\text { C5 } \\
\text { (I.V. } \\
\text { ) }\end{array}$ & Year* & $\begin{array}{c}\text { C3I } \\
\text { CB } \\
(E V)\end{array}$ & $\begin{array}{c}\text { C5I } \\
\text { CB } \\
(\mathrm{EV} \\
\text { ) }\end{array}$ & $\begin{array}{c}\text { C3 } \\
\text { ratio } \\
(\mathrm{RV})\end{array}$ & $\begin{array}{c}\text { C5 } \\
\text { ratio } \\
\text { (RV) }\end{array}$ & & \\
\hline $\begin{array}{c}1996 \\
(12) \\
\end{array}$ & $\begin{array}{c}146 . \\
34\end{array}$ & $\begin{array}{c}135 . \\
37\end{array}$ & 0.37 & 0.56 & $\begin{array}{c}0.2 \\
5\end{array}$ & 0.42 & $\begin{array}{c}1995- \\
1996 \\
(10) \\
\end{array}$ & $\begin{array}{c}161 . \\
11\end{array}$ & $\begin{array}{c}142 . \\
22\end{array}$ & 0.48 & 0.71 & $\begin{array}{c}0.3 \\
0\end{array}$ & $\begin{array}{c}0.5 \\
0\end{array}$ \\
\hline $\begin{array}{c}1997 \\
(12)\end{array}$ & $\begin{array}{c}150 . \\
60\end{array}$ & $\begin{array}{c}132 . \\
29\end{array}$ & 0.38 & 0.55 & $\begin{array}{c}0.2 \\
5\end{array}$ & 0.42 & $\begin{array}{c}1996- \\
1997 \\
(12) \\
\end{array}$ & $\begin{array}{c}148 . \\
48\end{array}$ & $\begin{array}{c}140 . \\
91\end{array}$ & 0.37 & 0.59 & $\begin{array}{c}0.2 \\
5\end{array}$ & $\begin{array}{c}0.4 \\
2\end{array}$ \\
\hline $\begin{array}{c}1998 \\
(12)\end{array}$ & $\begin{array}{c}142 . \\
86\end{array}$ & $\begin{array}{c}132 . \\
14\end{array}$ & 0.36 & 0.55 & $\begin{array}{c}0.2 \\
5\end{array}$ & 0.42 & $\begin{array}{c}1997- \\
1998 \\
(12) \\
\end{array}$ & $\begin{array}{c}153 . \\
03\end{array}$ & $\begin{array}{c}137 . \\
27\end{array}$ & 0.38 & 0.57 & $\begin{array}{c}0.2 \\
5\end{array}$ & $\begin{array}{c}0.4 \\
2\end{array}$ \\
\hline $\begin{array}{c}1999 \\
(12)\end{array}$ & $\begin{array}{c}135 . \\
02\end{array}$ & $\begin{array}{c}127 \\
19\end{array}$ & 0.34 & 0.53 & $\begin{array}{c}0.2 \\
5\end{array}$ & 0.42 & $\begin{array}{c}1998- \\
1999 \\
(14)\end{array}$ & $\begin{array}{c}147 . \\
44\end{array}$ & $\begin{array}{c}137 . \\
69\end{array}$ & 0.32 & 0.49 & $\begin{array}{c}0.2 \\
1\end{array}$ & $\begin{array}{c}0.3 \\
6\end{array}$ \\
\hline $\begin{array}{c}2000 \\
(12)\end{array}$ & $\begin{array}{c}139 . \\
34\end{array}$ & $\begin{array}{c}129 . \\
01\end{array}$ & 0.35 & 0.54 & $\begin{array}{c}0.2 \\
5\end{array}$ & 0.42 & $\begin{array}{c}1999- \\
2000 \\
(12)\end{array}$ & $\begin{array}{c}150 . \\
56\end{array}$ & $\begin{array}{c}138 . \\
88\end{array}$ & 0.38 & 0.58 & $\begin{array}{c}0.2 \\
5\end{array}$ & $\begin{array}{c}0.4 \\
2\end{array}$ \\
\hline $\begin{array}{c}2001 \\
(12)\end{array}$ & $\begin{array}{c}134 . \\
94\end{array}$ & $\begin{array}{c}125 . \\
06\end{array}$ & 0.34 & 0.52 & $\begin{array}{c}0.2 \\
5\end{array}$ & 0.42 & $\begin{array}{c}2000- \\
2001 \\
(12)\end{array}$ & $\begin{array}{c}143 . \\
49\end{array}$ & $\begin{array}{c}130 . \\
67\end{array}$ & 0.36 & 0.54 & $\begin{array}{c}0.2 \\
5\end{array}$ & $\begin{array}{c}0.4 \\
2\end{array}$ \\
\hline $\begin{array}{c}2002 \\
(12)\end{array}$ & $\begin{array}{c}153 . \\
75\end{array}$ & $\begin{array}{c}144 . \\
14\end{array}$ & 0.38 & 0.60 & $\begin{array}{c}0.2 \\
5\end{array}$ & 0.42 & $\begin{array}{c}2001- \\
2002 \\
(12) \\
\end{array}$ & $\begin{array}{c}141 . \\
25\end{array}$ & $\begin{array}{c}128 . \\
28\end{array}$ & 0.35 & 0.53 & $\begin{array}{c}0.2 \\
5\end{array}$ & $\begin{array}{c}0.4 \\
2\end{array}$ \\
\hline $\begin{array}{c}2003 \\
(12)\end{array}$ & $\begin{array}{c}145 . \\
03\end{array}$ & $\begin{array}{c}130 . \\
53\end{array}$ & 0.36 & 0.54 & $\begin{array}{c}0.2 \\
5\end{array}$ & 0.42 & $\begin{array}{c}2002- \\
2003 \\
(12) \\
\end{array}$ & $\begin{array}{c}135 . \\
81\end{array}$ & $\begin{array}{c}127 . \\
30\end{array}$ & 0.34 & 0.53 & $\begin{array}{c}0.2 \\
5\end{array}$ & $\begin{array}{c}0.4 \\
2\end{array}$ \\
\hline $\begin{array}{c}2004 \\
(12)\end{array}$ & $\begin{array}{c}125 . \\
88\end{array}$ & $\begin{array}{c}121 . \\
41\end{array}$ & 0.31 & 0.51 & $\begin{array}{c}0.2 \\
5\end{array}$ & 0.42 & $\begin{array}{c}2003- \\
2004 \\
(12) \\
\end{array}$ & $\begin{array}{c}142 . \\
77\end{array}$ & $\begin{array}{c}131 . \\
58\end{array}$ & 0.36 & 0.55 & $\begin{array}{c}0.2 \\
5\end{array}$ & $\begin{array}{c}0.4 \\
2\end{array}$ \\
\hline $\begin{array}{c}2005 \\
(12)\end{array}$ & $\begin{array}{c}156 . \\
42\end{array}$ & $\begin{array}{c}138 . \\
99\end{array}$ & 0.39 & 0.58 & $\begin{array}{c}0.2 \\
5\end{array}$ & 0.42 & $\begin{array}{c}2004- \\
2005 \\
(12) \\
\end{array}$ & $\begin{array}{c}135 \\
47\end{array}$ & $\begin{array}{c}125 . \\
59\end{array}$ & 0.34 & 0.52 & $\begin{array}{c}0.2 \\
5\end{array}$ & $\begin{array}{c}0.4 \\
2\end{array}$ \\
\hline $\begin{array}{c}2006 \\
(14)\end{array}$ & $\begin{array}{c}148 . \\
96\end{array}$ & $\begin{array}{c}136 . \\
88\end{array}$ & 0.32 & 0.49 & $\begin{array}{c}0.2 \\
1\end{array}$ & 0.36 & $\begin{array}{c}2005- \\
2006 \\
(12) \\
\end{array}$ & $\begin{array}{c}130 . \\
90\end{array}$ & $\begin{array}{c}123 . \\
99\end{array}$ & 0.33 & 0.52 & $\begin{array}{c}0.2 \\
5\end{array}$ & $\begin{array}{c}0.4 \\
2\end{array}$ \\
\hline $\begin{array}{r}2007 \\
(14)\end{array}$ & $\begin{array}{c}136 . \\
82\end{array}$ & $\begin{array}{c}134 . \\
27\end{array}$ & 0.29 & 0.48 & $\begin{array}{c}0.2 \\
1\end{array}$ & 0.36 & $\begin{array}{c}2006- \\
2007 \\
(12) \\
\end{array}$ & $\begin{array}{c}130 . \\
81\end{array}$ & $\begin{array}{c}125 . \\
50\end{array}$ & 0.33 & 0.52 & $\begin{array}{c}0.2 \\
5\end{array}$ & $\begin{array}{c}0.4 \\
2\end{array}$ \\
\hline $\begin{array}{c}2008 \\
(14)\end{array}$ & $\begin{array}{c}143 . \\
67\end{array}$ & $\begin{array}{c}137 . \\
80\end{array}$ & 0.31 & 0.49 & $\begin{array}{c}0.2 \\
1\end{array}$ & 0.36 & $\begin{array}{c}2007- \\
2008 \\
(12)\end{array}$ & $\begin{array}{c}134 . \\
38\end{array}$ & $\begin{array}{c}128 . \\
71\end{array}$ & 0.34 & 0.54 & $\begin{array}{c}0.2 \\
5\end{array}$ & $\begin{array}{c}0.4 \\
2\end{array}$ \\
\hline $\begin{array}{c}2009 \\
(14)\end{array}$ & $\begin{array}{c}134 \\
33\end{array}$ & $\begin{array}{c}129 . \\
55\end{array}$ & 0.29 & 0.46 & $\begin{array}{c}0.2 \\
1\end{array}$ & 0.36 & $\begin{array}{c}2008- \\
2009 \\
(12) \\
\end{array}$ & $\begin{array}{c}128 . \\
68\end{array}$ & $\begin{array}{c}125 . \\
52\end{array}$ & 0.32 & 0.52 & $\begin{array}{c}0.2 \\
5\end{array}$ & $\begin{array}{c}0.4 \\
2\end{array}$ \\
\hline $\begin{array}{c}2010 \\
(14)\end{array}$ & $\begin{array}{c}138 . \\
04\end{array}$ & $\begin{array}{c}132 . \\
27\end{array}$ & 0.30 & 0.47 & $\begin{array}{c}0.2 \\
1\end{array}$ & 0.36 & $\begin{array}{c}2009- \\
2010 \\
(12) \\
\end{array}$ & $\begin{array}{c}139 . \\
67\end{array}$ & $\begin{array}{c}130 . \\
23\end{array}$ & 0.35 & 0.54 & $\begin{array}{c}0.2 \\
5\end{array}$ & $\begin{array}{c}0.4 \\
2\end{array}$ \\
\hline
\end{tabular}


International Journal of Entrepreneurial Knowledge

Issue 1/2016, Volume 4

\begin{tabular}{|c|c|c|c|c|c|c|c|c|c|c|c|c|c|}
\hline $\begin{array}{c}2011 \\
(15)\end{array}$ & $\begin{array}{c}136 . \\
24\end{array}$ & $\begin{array}{c}131 . \\
04\end{array}$ & 0.27 & 0.44 & $\begin{array}{c}0.2 \\
0\end{array}$ & 0.33 & $\begin{array}{c}2010- \\
2011 \\
(12)\end{array}$ & $\begin{array}{c}142 . \\
58\end{array}$ & $\begin{array}{c}134 . \\
71\end{array}$ & 0.36 & 0.56 & $\begin{array}{c}0.2 \\
5\end{array}$ & $\begin{array}{c}0.4 \\
2\end{array}$ \\
\hline $\begin{array}{c}2012 \\
(15)\end{array}$ & $\begin{array}{c}130 . \\
37\end{array}$ & $\begin{array}{c}128 . \\
16\end{array}$ & 0.26 & 0.43 & $\begin{array}{c}0.2 \\
0\end{array}$ & 0.33 & $\begin{array}{c}2011- \\
2012 \\
(12) \\
\end{array}$ & $\begin{array}{c}140 . \\
95\end{array}$ & $\begin{array}{c}131 . \\
81\end{array}$ & 0.35 & 0.55 & $\begin{array}{c}0.2 \\
5\end{array}$ & $\begin{array}{c}0.4 \\
2\end{array}$ \\
\hline $\begin{array}{c}2013 \\
(15)\end{array}$ & $\begin{array}{c}133 . \\
10\end{array}$ & $\begin{array}{c}129 . \\
72\end{array}$ & 0.27 & 0.43 & $\begin{array}{c}0.2 \\
0\end{array}$ & 0.33 & $\begin{array}{c}2012- \\
2013 \\
(12)\end{array}$ & $\begin{array}{c}138 . \\
36\end{array}$ & $\begin{array}{c}130 . \\
19\end{array}$ & 0.35 & 0.54 & $\begin{array}{c}0.2 \\
5\end{array}$ & $\begin{array}{c}0.4 \\
2\end{array}$ \\
\hline $\begin{array}{c}2014 \\
(15)\end{array}$ & $\begin{array}{c}136 . \\
60\end{array}$ & $\begin{array}{c}127 . \\
84\end{array}$ & 0.27 & 0.43 & $\begin{array}{c}0.2 \\
0\end{array}$ & 0.33 & $\begin{array}{c}2013- \\
2014 \\
(12)\end{array}$ & $\begin{array}{c}148 . \\
91\end{array}$ & $\begin{array}{c}139 . \\
44\end{array}$ & 0.37 & 0.58 & $\begin{array}{c}0.2 \\
5\end{array}$ & $\begin{array}{c}0.4 \\
2\end{array}$ \\
\hline
\end{tabular}

*number in parenthesis show numbers of clubs in the league

Source: Author

Chart 1: C3ICB and C5ICB index in selected seasons for Super Rugby

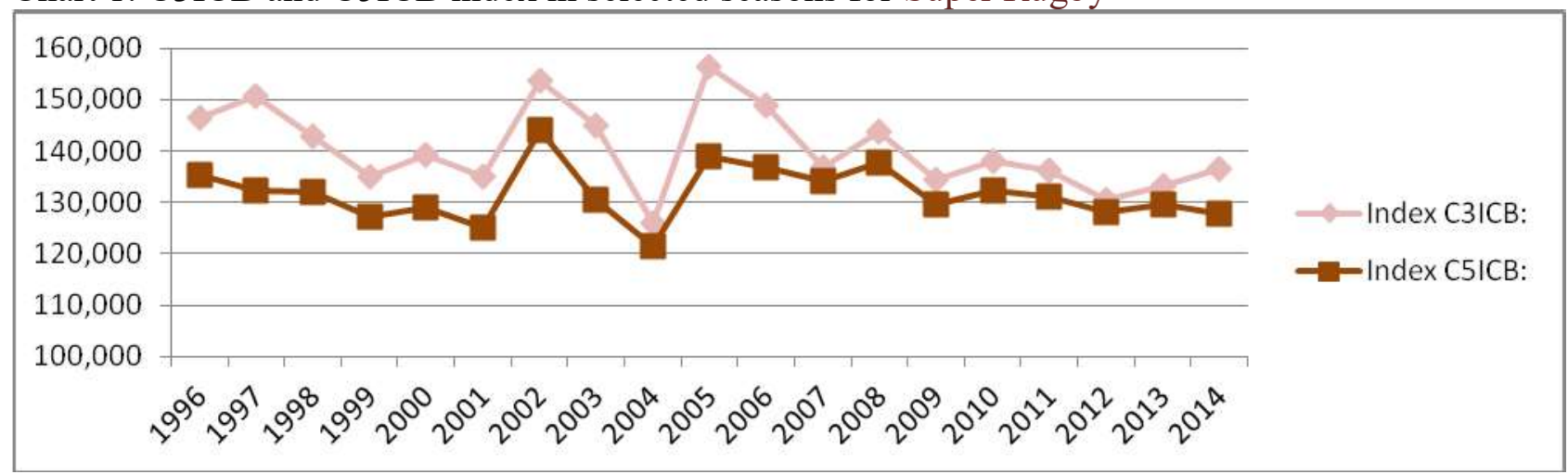

Source: Author

Chart 2: C3ICB and C5ICB index in selected seasons for Premiership League

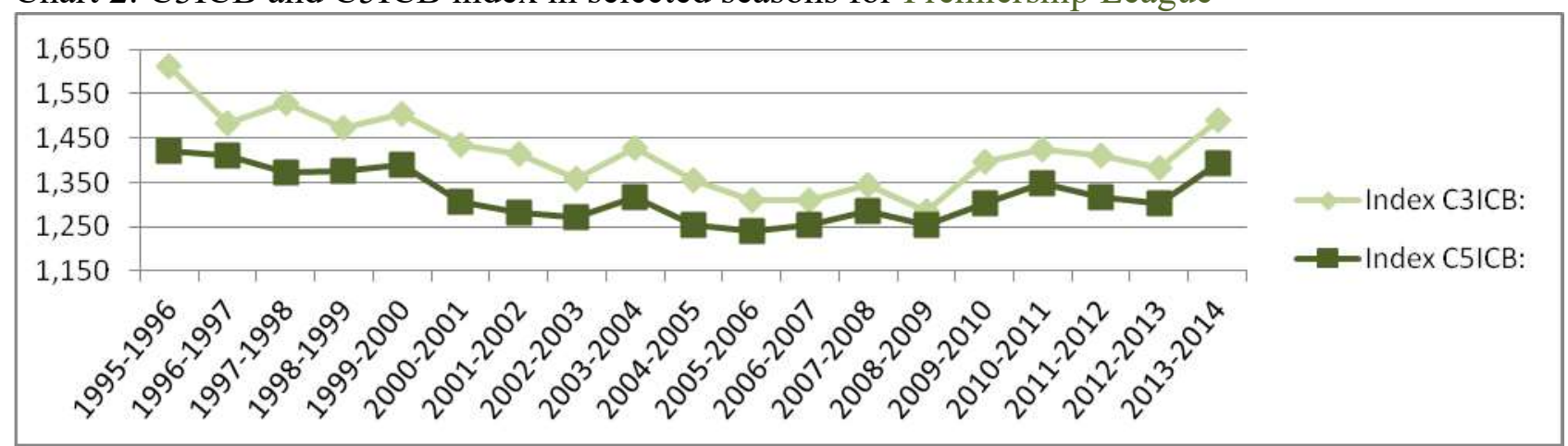

Source: Author

How we can see from the chart 1 the both index (C3ICB and C5ICB) in the case of Super Rugby has degreasing tendency from the year 2005, which is signalized, that the competitive balance is still better and better. The most balanced seasons was the year 2004, where both index s are at the minimums. It looks like the Super Rugby is on the right track every season.

Chart 2 showing us that competitive balance in Premiere League has worst results from the beginning, but after they introduced their Salary Cap in order (1999), the both index were degreasing. Till the season 2010 the competitive balance was one of the best in the rugby world. But last few seasons are dramatically growing, which is warning signal for English Rugby Union. Maybe the salary cap does not work anymore, hard to say wright now.

Table 2 plus charts 3 and 4 shows to us standard deviation of game results. That shows us how to win in the league diverted from the league average in the selected seasons. 
Table 2: standard deviation of game results in selected seasons

\begin{tabular}{|c|c|c|c|c|c|c|c|c|c|}
\hline \multicolumn{5}{|c|}{ Super Rugby League } & \multicolumn{5}{|c|}{ Premiership League } \\
\hline $\begin{array}{c}\text { Seaso } \\
\mathbf{n}\end{array}$ & ASD & $\begin{array}{c}\operatorname{ISD}(0,25 \\
)\end{array}$ & $\begin{array}{c}\text { ISD(seaso } \\
\text { n) }\end{array}$ & $\begin{array}{c}\text { ISD(His. } \\
\text { ) }\end{array}$ & $\begin{array}{c}\text { Seaso } \\
\mathrm{n}\end{array}$ & ASD & $\begin{array}{c}\operatorname{ISD}(0,25 \\
)\end{array}$ & $\begin{array}{c}\text { ISD(seaso } \\
\text { n) }\end{array}$ & $\begin{array}{c}\text { ISD(His. } \\
\text { ) }\end{array}$ \\
\hline 1996 & $\begin{array}{c}0.20 \\
0 \\
\end{array}$ & 0.131 & 0.150 & 0.131 & $\begin{array}{c}1995- \\
1996 \\
\end{array}$ & $\begin{array}{c}0.26 \\
8 \\
\end{array}$ & 0.102 & 0.117 & 0.043 \\
\hline 1997 & $\begin{array}{c}0.18 \\
6 \\
\end{array}$ & 0.131 & 0.146 & 0.131 & $\begin{array}{l}1996- \\
1997\end{array}$ & $\begin{array}{c}0.22 \\
8\end{array}$ & 0.092 & 0.105 & 0.043 \\
\hline 1998 & $\begin{array}{c}0.20 \\
6\end{array}$ & 0.131 & 0.150 & 0.131 & $\begin{array}{c}1997- \\
1998 \\
\end{array}$ & $\begin{array}{c}0.21 \\
8\end{array}$ & 0.092 & 0.105 & 0.043 \\
\hline 1999 & $\begin{array}{c}0.19 \\
7\end{array}$ & 0.131 & 0.147 & 0.131 & $\begin{array}{c}1998- \\
1999 \\
\end{array}$ & $\begin{array}{c}0.19 \\
9\end{array}$ & 0.085 & 0.097 & 0.043 \\
\hline 2000 & $\begin{array}{c}0.20 \\
8\end{array}$ & 0.131 & 0.148 & 0.131 & $\begin{array}{l}1999- \\
2000\end{array}$ & $\begin{array}{c}0.22 \\
2\end{array}$ & 0.092 & 0.105 & 0.043 \\
\hline 2001 & $\begin{array}{c}0.15 \\
7\end{array}$ & 0.131 & 0.151 & 0.131 & $\begin{array}{l}2000- \\
2001\end{array}$ & $\begin{array}{c}0.19 \\
5\end{array}$ & 0.092 & 0.106 & 0.043 \\
\hline 2002 & $\begin{array}{c}0.27 \\
8\end{array}$ & 0.131 & 0.151 & 0.131 & $\begin{array}{l}2001- \\
2002\end{array}$ & $\begin{array}{c}0.17 \\
2\end{array}$ & 0.092 & 0.105 & 0.043 \\
\hline 2003 & $\begin{array}{c}0.21 \\
4 \\
\end{array}$ & 0.131 & 0.151 & 0.131 & $\begin{array}{l}2002- \\
2003 \\
\end{array}$ & $\begin{array}{c}0.15 \\
0 \\
\end{array}$ & 0.092 & 0.104 & 0.043 \\
\hline 2004 & $\begin{array}{c}0.16 \\
7\end{array}$ & 0.131 & 0.148 & 0.131 & $\begin{array}{c}2003- \\
2004\end{array}$ & $\begin{array}{c}0.21 \\
9\end{array}$ & 0.092 & 0.104 & 0.043 \\
\hline 2005 & $\begin{array}{c}0.23 \\
1\end{array}$ & 0.128 & 0.144 & 0.131 & $\begin{array}{l}2004- \\
2005\end{array}$ & $\begin{array}{c}0.14 \\
4\end{array}$ & 0.092 & 0.104 & 0.043 \\
\hline 2006 & $\begin{array}{c}0.21 \\
2 \\
\end{array}$ & 0.120 & 0.136 & 0.131 & $\begin{array}{l}2005- \\
2006 \\
\end{array}$ & $\begin{array}{c}0.15 \\
1 \\
\end{array}$ & 0.092 & 0.104 & 0.043 \\
\hline 2007 & $\begin{array}{c}0.18 \\
2\end{array}$ & 0.120 & 0.137 & 0.131 & $\begin{array}{l}2006- \\
2007\end{array}$ & $\begin{array}{c}0.14 \\
5\end{array}$ & 0.092 & 0.104 & 0.043 \\
\hline 2008 & $\begin{array}{c}0.23 \\
1\end{array}$ & 0.120 & 0.136 & 0.131 & $\begin{array}{l}2007- \\
2008\end{array}$ & $\begin{array}{c}0.18 \\
5\end{array}$ & 0.092 & 0.105 & 0.043 \\
\hline 2009 & $\begin{array}{c}0.20 \\
1\end{array}$ & 0.120 & 0.138 & 0.131 & $\begin{array}{c}2008- \\
2009\end{array}$ & $\begin{array}{c}0.16 \\
0\end{array}$ & 0.092 & 0.105 & 0.043 \\
\hline 2010 & $\begin{array}{c}0.21 \\
4\end{array}$ & 0.120 & 0.137 & 0.131 & $\begin{array}{l}2009- \\
2010\end{array}$ & $\begin{array}{c}0.16 \\
9\end{array}$ & 0.092 & 0.102 & 0.043 \\
\hline 2011 & $\begin{array}{c}0.19 \\
9\end{array}$ & 0.106 & 0.120 & 0.131 & $\begin{array}{l}2010- \\
2011\end{array}$ & $\begin{array}{c}0.20 \\
4\end{array}$ & 0.092 & 0.105 & 0.043 \\
\hline 2012 & $\begin{array}{c}0.23 \\
3\end{array}$ & 0.108 & 0.125 & 0.131 & $\begin{array}{l}2011- \\
2012\end{array}$ & $\begin{array}{c}0.18 \\
1\end{array}$ & 0.092 & 0.105 & 0.043 \\
\hline 2013 & $\begin{array}{c}0.19 \\
7\end{array}$ & 0.108 & 0.123 & 0.131 & $\begin{array}{l}2012- \\
2013\end{array}$ & $\begin{array}{c}0.18 \\
7\end{array}$ & 0.092 & 0.105 & 0.043 \\
\hline 2014 & $\begin{array}{c}0.15 \\
0\end{array}$ & 0.108 & 0.124 & 0.131 & $\begin{array}{c}2013- \\
2014\end{array}$ & $\begin{array}{c}0.25 \\
1\end{array}$ & 0.092 & 0.105 & 0.043 \\
\hline
\end{tabular}

Source: Author

ASD -calculate winning \% as (\# of wins * $2+$ \# of ties)/(\# of games played * 2), and then Stdev.s ISD $(0,25)-[(1-p) / 4 n] 1 / 2$, where $p$ is the probability of a tie under the equal playing strength assumption and $n$ is the number of games played. In this case $p=0,25$

ISD (season) - [(1-p)/4n]1/2, where $p$ is the probability of a tie under the equal playing strength assumption and $n$ is the number of games played. In this case $p$ is calculated for every each one seasons.

ISD (His.) - [(1-p)/4n]1/2, where $p$ is the probability of a tie under the equal playing strength assumption and $\mathrm{n}$ is the number of games played. In this case $\mathrm{p}$ is calculated like an average from season 1996 to 2014. 
Chart 3: standard deviation of game results and ideal standard deviation in selected seasons for Super Rugby

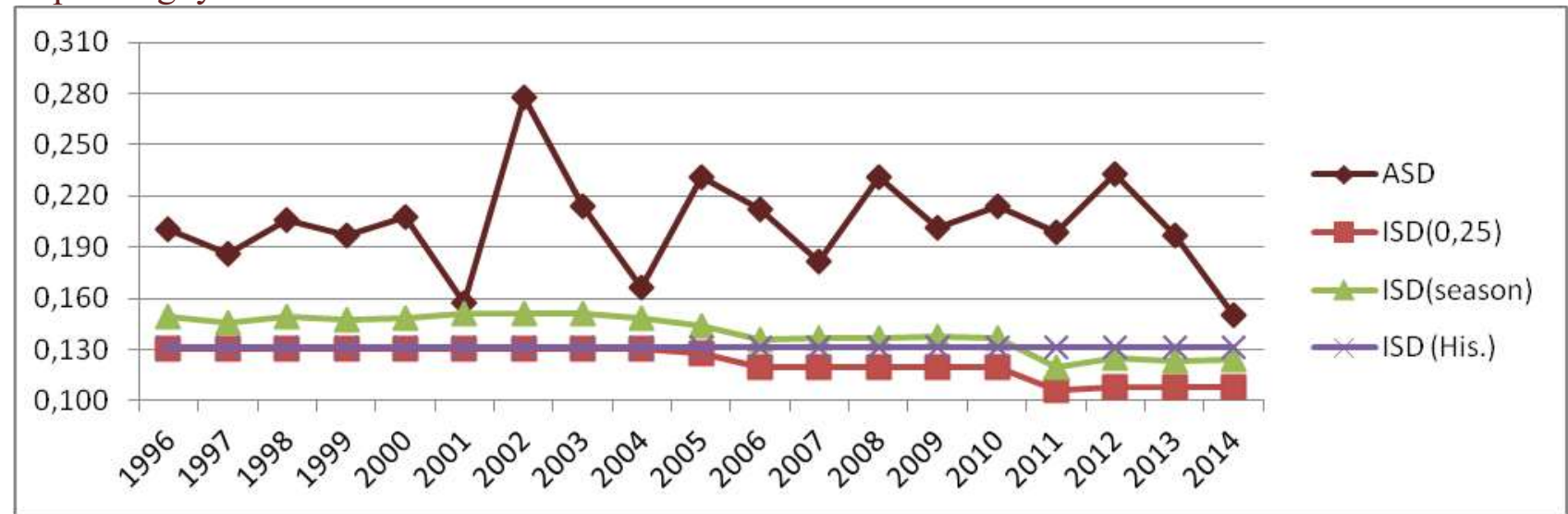

Source: Author

How we can see from the chart 2 the Actual standard deviation is mostly flowing between the range 0.190 and 0.230 , which is not bat at all. Even two times in the history (2001 and 2004) the ASD met the ISD. Does not matter if we are looking at ideal standard deviation of historical value or season value or with probability of $p=0,25$. The most extreme season was 2002 were Crusader won every match and also they became champions without losing any point. On the other hand the Bulls and Lions were defeated every time. That was only one time in history were the club won the league without losing any point and also there was the biggest different between the first and second position in history (12points). From the season 2012 is standard deviation of game results degreasing rapidly and the last played season almost hit the goal again. Maybe it is caused, because the league has more teams so the winner points are split or divided more broadly. It will be interesting to see what will be happened to next season.

Chart 4: standard deviation victories and ideal standard deviation in selected seasons for Premiership League

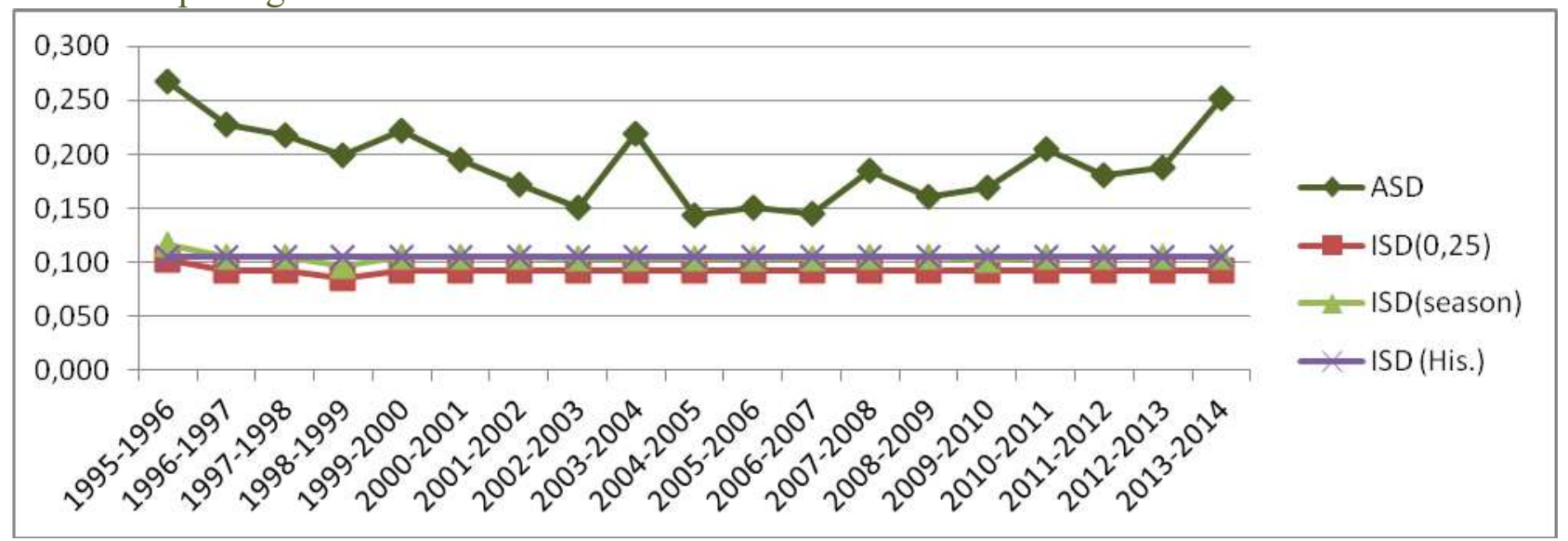

Source: Author

The English Premiership League are more constant than Super Rugby, but the actual standard deviation is addicted on the numbers of team in the season, so that is the reason why is Super Rugby more changeable. From the year when they introduced the salary cap were ASD very close to ISD. Only the season 2003-2004 had more significant change. It happened because Rotherham Titans lost all 22 games. This was only second time in history that the team could not win any game. First time it was in first observed season (1995-1996) with the team West Hartlepool. 
Table 3 and charts shows relative standard deviation - the ratio of actual and ideal standard deviation extends the previous indicator of relationship to the situation which would occur in ideal distribution victories. Is often attributed to Noll (1988) and Scully (1989). How mention about the ASD was is addicted on numbers of teams in the seasons, so in this case is better to use Relative standard deviation.

Table 3: relative standard deviation

\begin{tabular}{|c|c|c|c|}
\hline \multicolumn{4}{|c|}{ Super Rugby League } \\
\hline Season & $\begin{array}{c}\text { RSD } \\
\text { (0,25) }\end{array}$ & $\begin{array}{c}\text { RSD } \\
\text { (season) }\end{array}$ & $\begin{array}{c}\text { RSD } \\
\text { (His.) }\end{array}$ \\
\hline $\mathbf{1 9 9 6}$ & 1.536 & 1.340 & 1.527 \\
\hline $\mathbf{1 9 9 7}$ & 1.424 & 1.272 & 1.416 \\
\hline $\mathbf{1 9 9 8}$ & 1.578 & 1.377 & 1.569 \\
\hline $\mathbf{1 9 9 9}$ & 1.507 & 1.335 & 1.498 \\
\hline $\mathbf{2 0 0 0}$ & 1.592 & 1.400 & 1.583 \\
\hline $\mathbf{2 0 0 1}$ & 1.206 & 1.044 & 1.199 \\
\hline $\mathbf{2 0 0 2}$ & 2.131 & 1.845 & 2.118 \\
\hline $\mathbf{2 0 0 3}$ & 1.640 & 1.420 & 1.630 \\
\hline $\mathbf{2 0 0 4}$ & 1.277 & 1.123 & 1.270 \\
\hline $\mathbf{2 0 0 5}$ & 1.806 & 1.599 & 1.756 \\
\hline $\mathbf{2 0 0 6}$ & 1.768 & 1.566 & 1.617 \\
\hline $\mathbf{2 0 0 7}$ & 1.513 & 1.325 & 1.383 \\
\hline $\mathbf{2 0 0 8}$ & 1.922 & 1.692 & 1.757 \\
\hline $\mathbf{2 0 0 9}$ & 1.676 & 1.459 & 1.533 \\
\hline $\mathbf{2 0 1 0}$ & 1.781 & 1.560 & 1.629 \\
\hline $\mathbf{2 0 1 1}$ & 1.874 & 1.656 & 1.514 \\
\hline $\mathbf{2 0 1 2}$ & 2.149 & 1.861 & 1.772 \\
\hline $\mathbf{2 0 1 3}$ & 1.816 & 1.593 & 1.497 \\
\hline $\mathbf{2 0 1 4}$ & 1.384 & 1.209 & 1.141 \\
\hline
\end{tabular}

\begin{tabular}{|c|c|c|c|}
\hline \multicolumn{4}{|c|}{ Premiership League } \\
\hline Season & $\begin{array}{c}\text { RSD } \\
\text { (0,25) }\end{array}$ & $\begin{array}{c}\text { RSD } \\
\text { (season) }\end{array}$ & $\begin{array}{c}\text { RSD } \\
\text { (His.) }\end{array}$ \\
\hline $\mathbf{1 9 9 5 - 1 9 9 6}$ & 2.626 & 2.287 & 2.558 \\
\hline $\mathbf{1 9 9 6 - 1 9 9 7}$ & 2.469 & 2.171 & 2.175 \\
\hline $\mathbf{1 9 9 7 - 1 9 9 8}$ & 2.361 & 2.077 & 2.080 \\
\hline $\mathbf{1 9 9 8 - 1 9 9 9}$ & 2.338 & 2.048 & 1.895 \\
\hline $\mathbf{1 9 9 9 - 2 0 0 0}$ & 2.408 & 2.117 & 2.121 \\
\hline $\mathbf{2 0 0 0 - 2 0 0 1}$ & 2.110 & 1.841 & 1.859 \\
\hline $\mathbf{2 0 0 1 - 2 0 0 2}$ & 1.863 & 1.645 & 1.641 \\
\hline $\mathbf{2 0 0 2 - 2 0 0 3}$ & 1.630 & 1.444 & 1.436 \\
\hline $\mathbf{2 0 0 3 - 2 0 0 4}$ & 2.368 & 2.108 & 2.087 \\
\hline $\mathbf{2 0 0 4 - 2 0 0 5}$ & 1.557 & 1.380 & 1.372 \\
\hline $\mathbf{2 0 0 5 - 2 0 0 6}$ & 1.636 & 1.456 & 1.442 \\
\hline $\mathbf{2 0 0 6 - 2 0 0 7}$ & 1.575 & 1.396 & 1.387 \\
\hline $\mathbf{2 0 0 7 - 2 0 0 8}$ & 2.006 & 1.757 & 1.767 \\
\hline $\mathbf{2 0 0 8 - 2 0 0 9}$ & 1.734 & 1.531 & 1.528 \\
\hline $\mathbf{2 0 0 9 - 2 0 1 0}$ & 1.833 & 1.651 & 1.615 \\
\hline $\mathbf{2 0 1 0 - 2 0 1 1}$ & 2.214 & 1.947 & 1.951 \\
\hline $\mathbf{2 0 1 1 - 2 0 1 2}$ & 1.955 & 1.720 & 1.723 \\
\hline $\mathbf{2 0 1 2 - 2 0 1 3}$ & 2.030 & 1.785 & 1.789 \\
\hline $\mathbf{2 0 1 3 - 2 0 1 4}$ & 2.721 & 2.384 & 2.397 \\
\hline
\end{tabular}

$*$ Relative standard deviation is calculating $=\mathrm{ASD} / \mathrm{ISD}$

Source: Author

Chart 5: relative standard deviation for Super Rugby

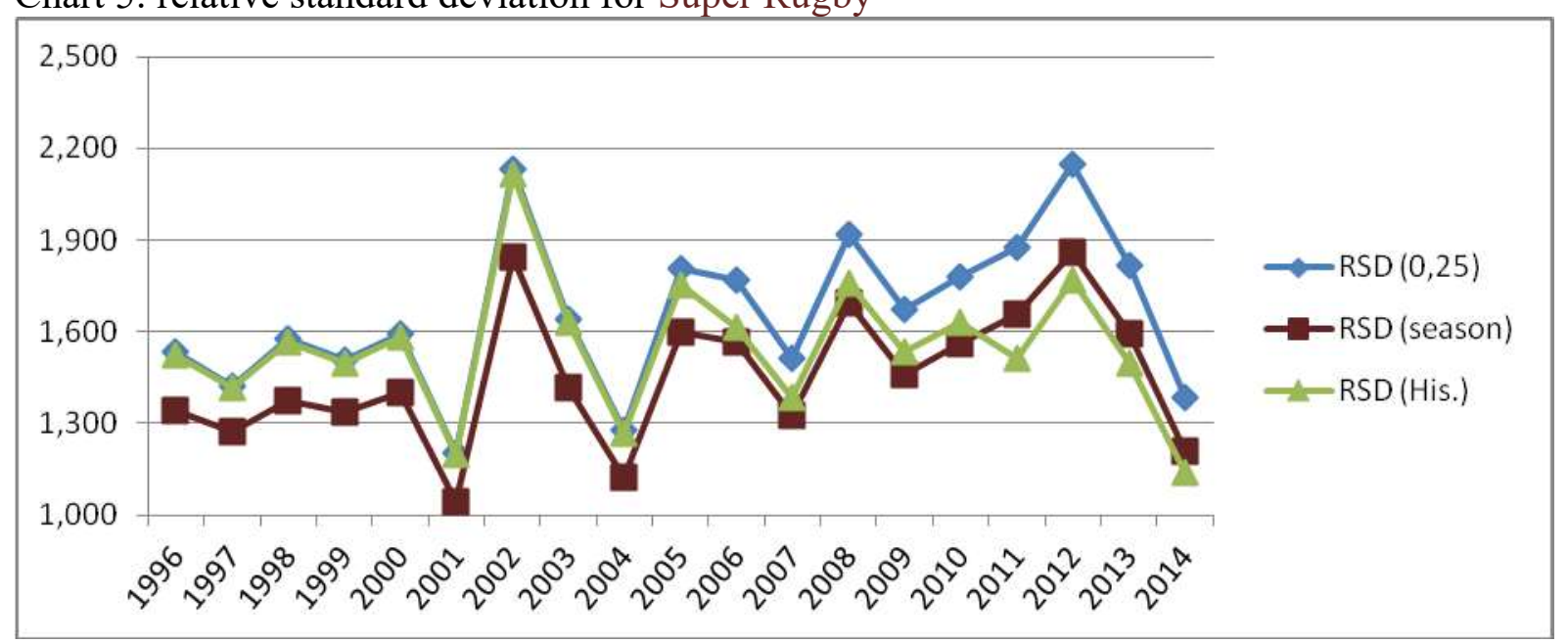

Source: Author

How we can see from the chart the relative standard deviation is clearly much higher than the ideal standard deviation. It is almost two times higher. We can approximately say, that all three types of RSD are flowing between the ranges from 1,3 to 1,9 most of the times. Most important is RSD (season), because it is most reflecting one the changes during the period. 
Chart 6: relative standard deviation for Premiership League

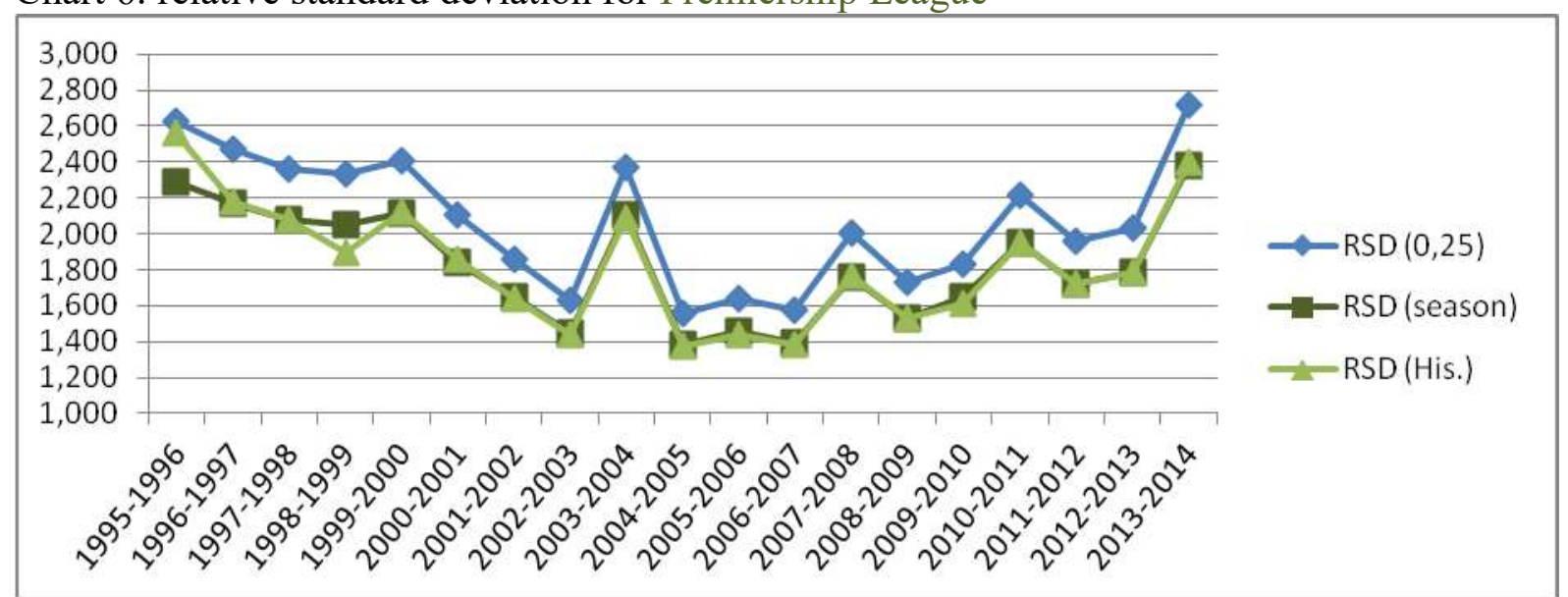

Source: Author

How we can see from the chart the Premiere League has relative standard deviation higher than the Super Rugby. Most of the time is flowing between the range 1.500 and 2.000. And the last observed season had the worst result ever for the RSD season. We can clearly say, that last three seasons, are still worst and worst.

Charts 7,8,9 and 10 shows Number of winners, respectively frequency of champions examines how often changing over time teams to master position. But we still can take a look on this model from two different sides. First one is regular season and who became a champions and the other one is play of part. It can be different result, because the winner of regular season could not be a winner of play of. Next four charts are showing the differences in both cases. 
Chart 7: number of winners in regular seasons for Super Rugby

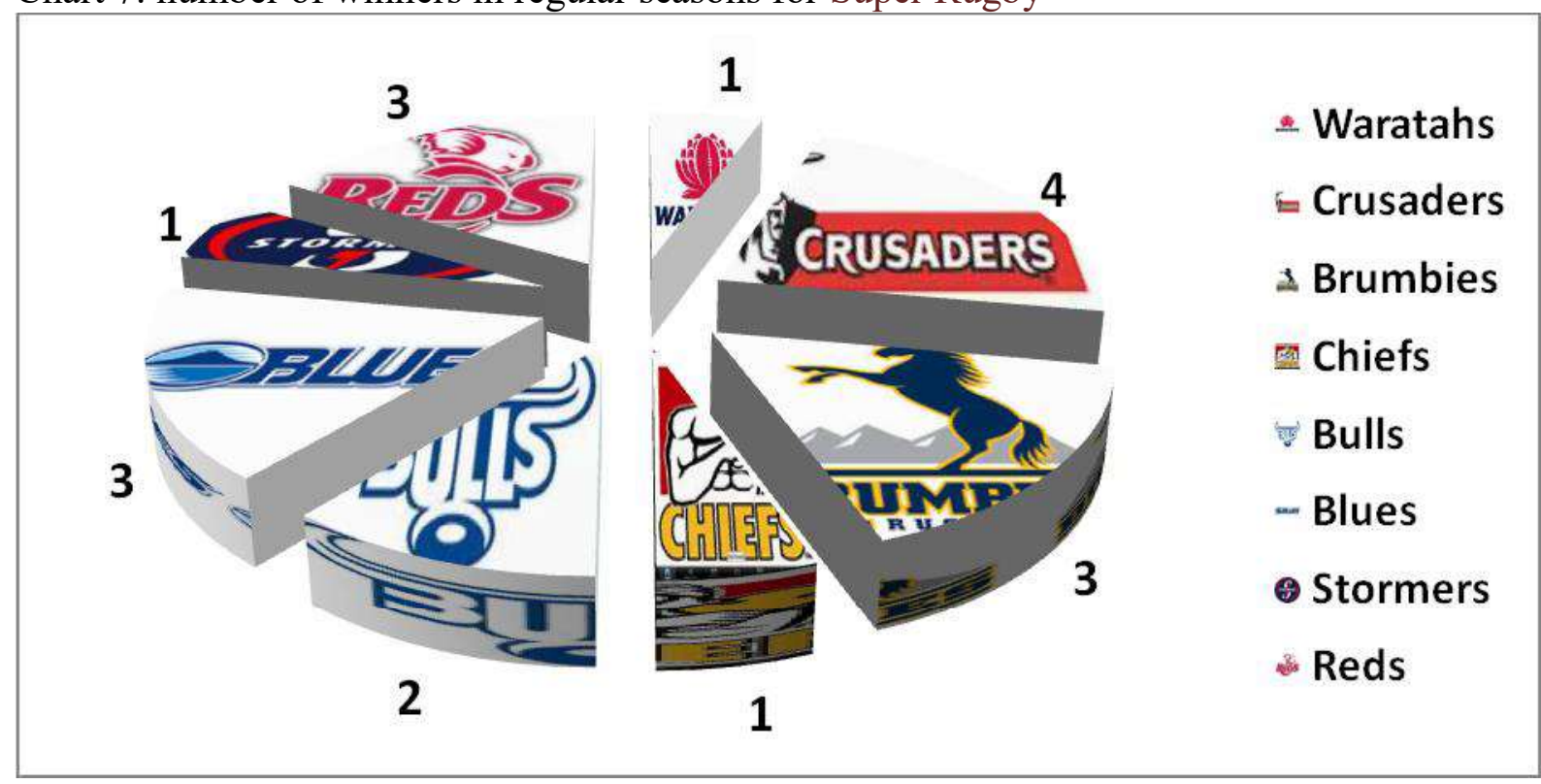

Source: Author

How we can see this model bring to us little bit different result, as the others one before. The league from the models used before signalized, that is it not very balanced. But this model shows us, that in the last nineteen regular seasons the league had eight different winners from fifteen teams. Which is pretty good result. It is hard to predict who will be won the league. Let's take a look on play off chart.

Chart 8: number of winners in play off for Super Rugby

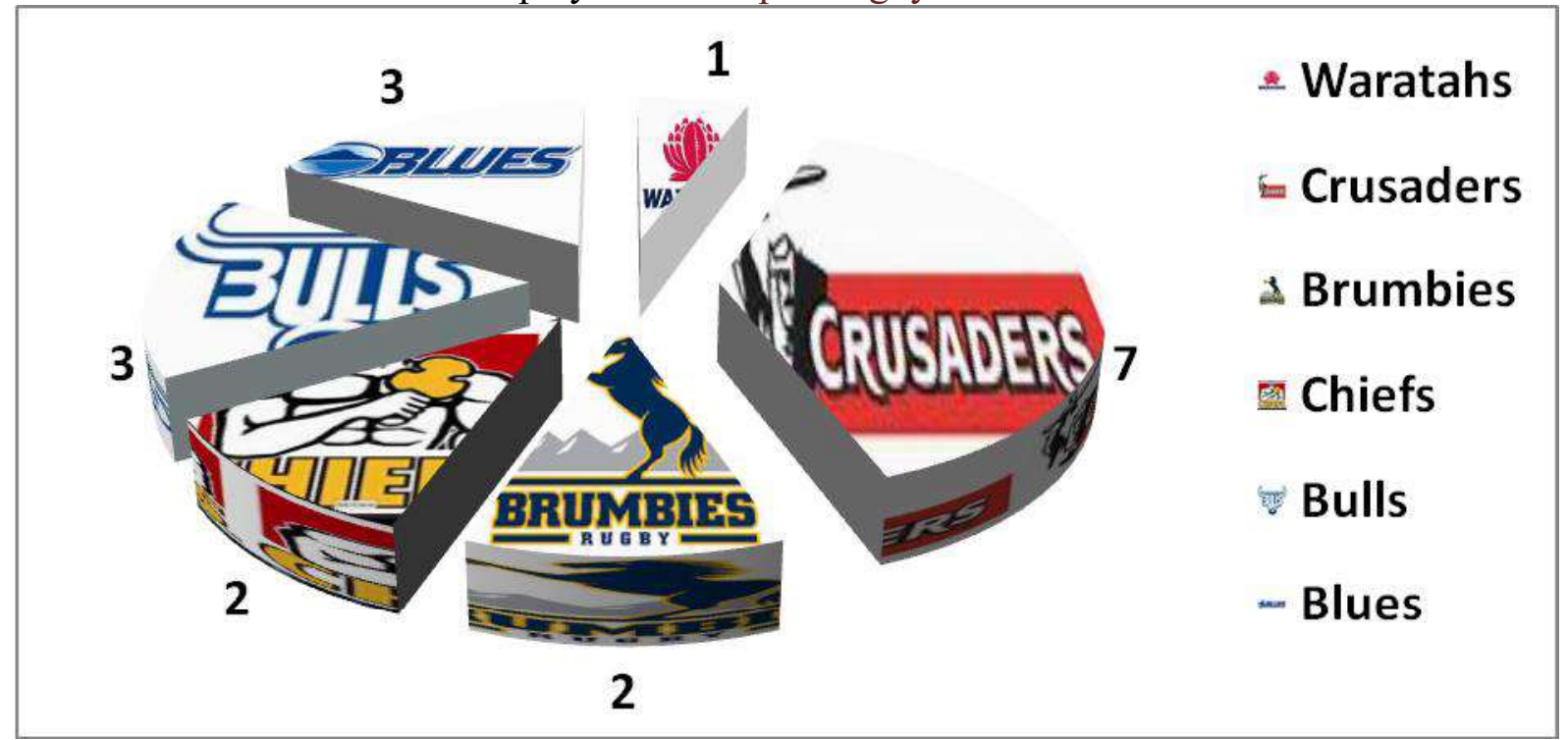

Source: Author

The play off chart has little bit different result than one before (regular season). The numbers of winner are two less and there is a stronger domination of team Crusaders. 
Chart 9: number of winners in regular seasons for Premiership League

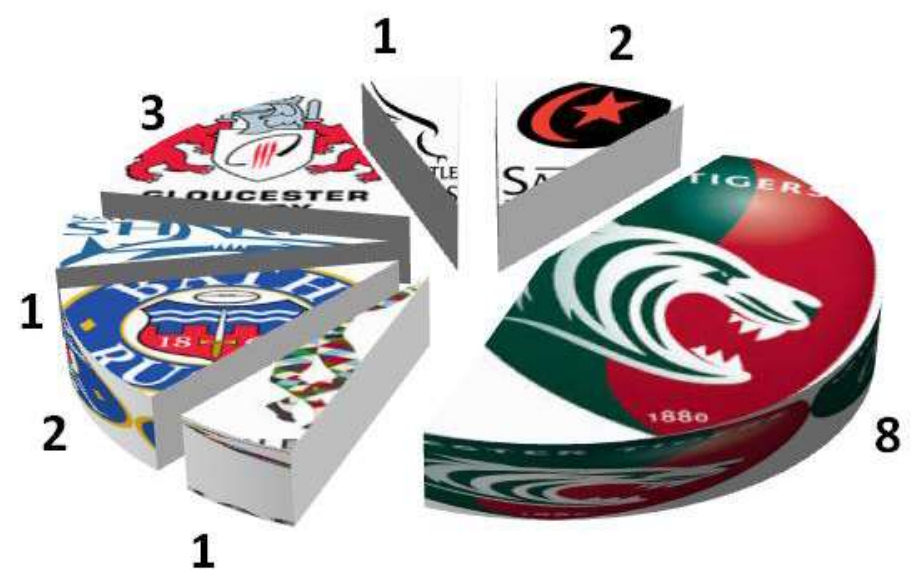

Saracens

Leicester Tigers

Harlequins

Bath Rugby

Sale Sharks

Gloucester Rugby

Newcastle Falcons

\section{Source: Author}

The result from the regular seasons charts shows that during the nineteenth observed seasons were seven different winners from seventeen teams. But we also can see the dominations of Leicester Tigers, which won eight times. So it not very competition balanced. Let's take a look on play off chart.

Chart 10: number of winners in play off for Premiership League

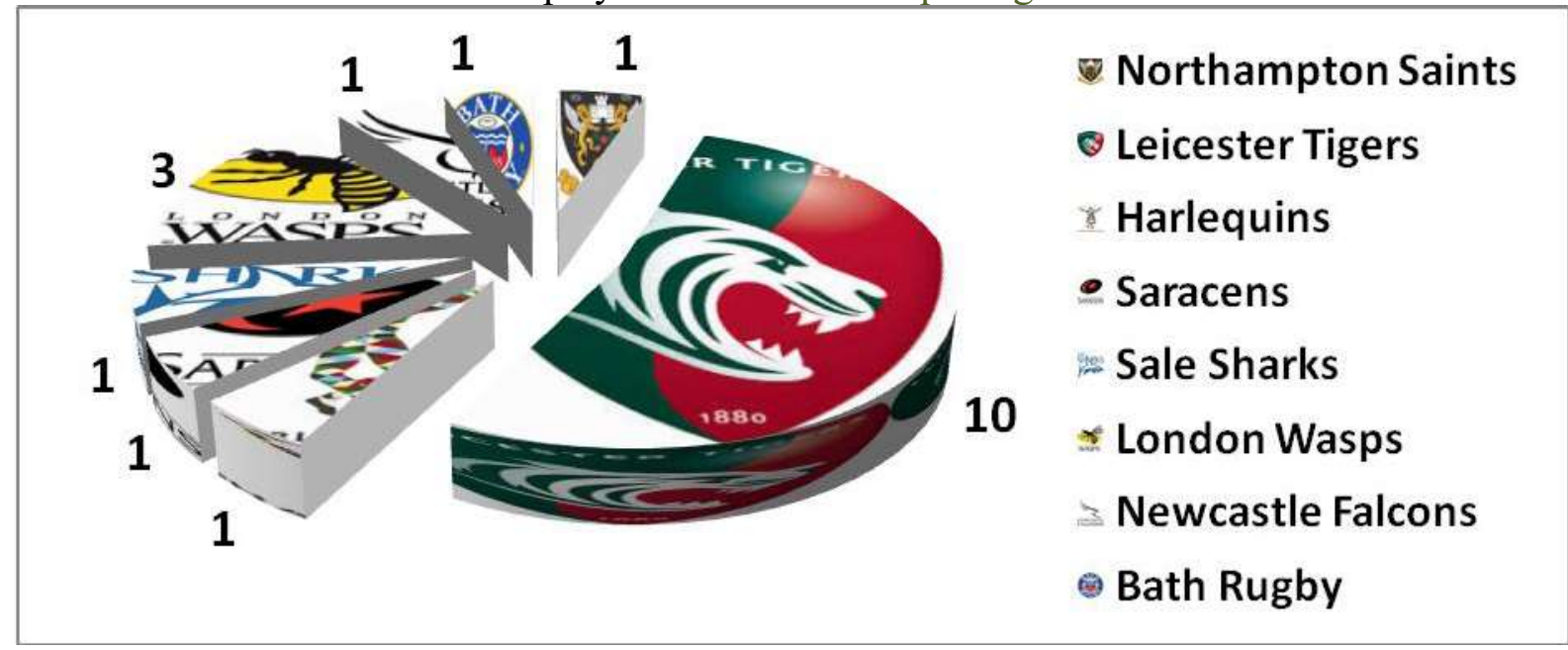

Source: Author

How we can see, there is opposite effect than in the Super Rugby. The numbers of winner in play off part are bigger than in the regular season, truly from eight to ten teams. But the dominations of Leicester Tigers are even bigger, because they won ten times from nineteen possibilities. The salary cap did not bring the difference to this model.

Table 5 and 6 shows a placement in the k-th positions is the latest way of measuring the competitive equilibrium, which is used in this paper. We are interested not only who is at the first place, but how many teams are placed in positions in the second, third, fourth, respectively $\mathrm{k}$-th positions. It is interesting from the reason that the first four teams are playing the play off. 
Table 5: placement in the k-th positions for Super Rugby

\begin{tabular}{|c|c|c|c|c|c|}
\hline \multicolumn{7}{|c|}{ Placement in the k-th positions from 1996-2014 } \\
\hline & first & second & third & fourth & $\begin{array}{c}\text { Together to } \\
\mathbf{4} .\end{array}$ \\
\hline Waratahs & 1 & 3 & 2 & 1 & $\mathbf{7}$ \\
\hline Crusaders & 4 & 7 & 0 & 4 & $\mathbf{1 5}$ \\
\hline Sharks & 0 & 1 & 3 & 2 & $\mathbf{6}$ \\
\hline Brumbies & 3 & 1 & 2 & 2 & $\mathbf{8}$ \\
\hline Chiefs & 1 & 2 & 0 & 1 & $\mathbf{4}$ \\
\hline Highlanders & 0 & 0 & 2 & 2 & $\mathbf{4}$ \\
\hline Hurricanes & 0 & 1 & 3 & 2 & $\mathbf{6}$ \\
\hline Force & 0 & 0 & 0 & 0 & $\mathbf{0}$ \\
\hline Bulls & 2 & 1 & 3 & 0 & $\mathbf{6}$ \\
\hline Blues & 3 & 1 & 0 & 2 & $\mathbf{6}$ \\
\hline Stormers & 1 & 2 & 1 & 0 & $\mathbf{4}$ \\
\hline Lions & 0 & 0 & 1 & 1 & $\mathbf{2}$ \\
\hline Reds & 3 & 0 & 1 & 1 & $\mathbf{5}$ \\
\hline Cheetahs & 0 & 0 & 1 & 0 & $\mathbf{1}$ \\
\hline Rebels & 0 & 0 & 0 & 0 & $\mathbf{0}$ \\
\hline Cumulative & $\mathbf{8}$ & $\mathbf{1 0}$ & $\mathbf{1 3}$ & $\mathbf{1 3}$ & \\
\hline Kings are not counted-played only one season & & & \\
\hline
\end{tabular}

Source: Author

How we can see from the last used model placement in the k-th positions, the top two it is not so much changeable. Cumulative result shows us, that most of the seasons are first two places are occupant by the same clubs. It is only one or two different clubs per season, which are substitutes the strongest one. But the in the top four during the selected observed period were almost every each one team, instead of three of them. So it means that almost every team in the league had a change to won the title in play-off.

Table 6: placement in the k-th positions for Premiership League

\begin{tabular}{|c|c|c|c|c|c|}
\hline \multicolumn{7}{|c|}{ Placement in the k-th positions from 1996-2014 } \\
\hline Team & first & second & third & fourth & $\begin{array}{c}\text { together to } \\
\text { 4. }\end{array}$ \\
\hline Saracens & 2 & 2 & 3 & 2 & $\mathbf{9}$ \\
\hline Northampton Saints & 0 & 3 & 2 & 4 & 9 \\
\hline Leicester Tigers & 8 & 5 & 2 & 3 & $\mathbf{1 8}$ \\
\hline Harlequins & 1 & 1 & 3 & 2 & 7 \\
\hline Bath Rugby & 2 & 2 & 3 & 3 & $\mathbf{1 0}$ \\
\hline Sale Sharks & 1 & 1 & 1 & 0 & $\mathbf{3}$ \\
\hline London Wasps & 1 & 5 & 0 & 2 & $\mathbf{8}$ \\
\hline Exeter Chiefs & 0 & 0 & 0 & 0 & $\mathbf{0}$ \\
\hline Gloucester Rugby & 3 & 0 & 3 & 1 & $\mathbf{7}$ \\
\hline London Irish & 0 & 0 & 2 & 1 & $\mathbf{3}$ \\
\hline Newcastle Falcons & 1 & 0 & 0 & 0 & $\mathbf{1}$ \\
\hline Worcester Warriors & 0 & 0 & 0 & 0 & $\mathbf{0}$ \\
\hline Bristol Shoguns & 0 & 0 & 1 & 0 & $\mathbf{1}$ \\
\hline Richmond & 0 & 0 & 0 & 0 & $\mathbf{0}$ \\
\hline Leeds Tykes & 0 & 0 & 0 & 1 & $\mathbf{1}$ \\
\hline Rotherham Titans & 0 & 0 & 0 & 0 & $\mathbf{0}$ \\
\hline
\end{tabular}




\begin{tabular}{|c|c|c|c|c|c|}
\hline Bedford Blues & 0 & 0 & 0 & 0 & $\mathbf{0}$ \\
\hline cumulative & $\mathbf{8}$ & $\mathbf{9}$ & $\mathbf{1 1}$ & $\mathbf{1 2}$ & \\
\hline London Scottish played only one seasons - no counted \\
\hline West Hartlepool \\
\hline
\end{tabular}

Source: Author

How we can see from the table 6 the Premiership has the same cumulative result as Super Rugby on the first position. However the second, third and the fourth are not growing so much from the position to position. That is reflecting worst competitive balance. From the seventeen teams five of them never played play off and even three of them was in best four once (on the third and fourth position). Only once the Leicester Tigers miss the play-off games. It is very strong domination from this team, and other three - five more. The rest are lucky if they had change to take a look in playoff games.

\section{DISCUSSION}

The issue of the paper was to compare two different rugby leagues in the nineteen seasons period. It starts from the year 1996 to the last played season 2014. The paper used the secondary data and results from the league standings. One League is from Europe - English Premiere League, where they had applied salary cap in the 1999 in order and the second one is Super Rugby which is consist of three different nations (Australia, South Africa and New Zealand). The main issue was took a look on competitive balance in those leagues, because they are working on the different principles. In the Super Rugby, there are now restrictions, only the money gates sharing, but the league in the observed period grow from original 12 teams to 15 and there are still some discussion about next growing. So the first issue was take a look if is it helpful for competitive balance to involved still more and more teams to the league. On the other hand there was English Premiere League with salary cap restriction. Salary cap is one of the most powerful restriction, because, the most strongest and richest clubs can spend on salary the same sum of money as the weakest, so they do not have a change to collect all the best players, because they cannot pay them the salary as they are worthy. The salary cap comes from the US leagues. But the salary cap is only one of the restrictions. In USA, they have also the draft, money sharing, luxury tax and licensing model. So it is more compact. The second issue was to take a look how the salary cap helps to bring competitive balance in the Premiere league. And on the end compere these two results. In the paper were used four different models to measuring the competitive balance. Each one is looking at competitive balance from a different point of view.

\section{CONCLUSIONS}

The result of the first issue (competitive balance in Super Rugby) is that the involving the more clubs into the league is a good choice for them. From the beginning of the observed period the league were little bit more competitive balanced, how we can see from the RSD in the chart 5, but last few years there is strong decline in the curve. Also last five years the league had every season different champions. All models are showing that the Super Rugby is on the right track. It will be interesting to continue in this research after few more seasons. And also could be interesting to compare with the effect on the average attendance. This additional research can bring interesting outcome for potential sponsors and company whose are thinking about sponsorship and propagating himself. Plus recently were the world cup in rugby in final Australia and New Zealand what can have a strong influence on attendance and money in rugby in those countries, so we can expect also some changes in competitive balance after few years. 
The result of the second issue (competitive balance in Premiership League) is that after they had involved the salary cap the RSD was much better than before. But last few years look like the salary cap stops working. The curve has increasing tendency and output is reaching the worst result from the observed period. The others models are also against that the salary cap is working. From the outputs we can see, that there is a domination of the team Leicester Tigers, even after the league involved the salary cap. Only one positive result is that the last four seasons has four different champions. But still the league is constantly spinning around few teams in final four.

The result of the third issue (comparison of competitive balance between the leagues) show, that better result comes out from the Super Rugby League than from the Premiership League. The Super Rugby won in almost every each one models. Only in the numbers of play-off winners are little bit worse than Premiership League. So we can clearly say that the strategy of involving the more clubs to the league is better than salary cap. That is does not mean the salary cap is not effective, but maybe it needs to be supported by others restrictions, like a drafts or something else. Maybe it can be much more worthy to think about make a connection with France Rugby League Championship 14 , but this is just author idea.

Next few studies will be about compering the competitive balance with the average attendance and the link between those inputs and also to compere competitive balance with other rugby leagues in the world.

\section{REFERENCES}

1. ANDREFF, Wladimir, SZYMANSKI, Stefan, Handbook of the Economics of Sport. 1.vyd. Cheltenham: Edward Elgar Publishing Limited, 2006. ISBN 1-84376-608-6

2. BRUNELLI M. The impact of UEFA Champions League upon domestic championships: the Italian Serie A. Nyon: UEFA/IASE Seminar; May 2nd, 2006.

3. DABSCHECK, B. (2004). Industrial Relations in Australasian Professional Team Sports. Otemon Journal of Australian Studies, 30 (4), 13-17.

4. DEJONGHE T. The evolution of Belgian football over the last decades. Nyon: UEFA/IASE Seminar; May 2nd, 2006.

5. FEDDERSEN A. Economic consequences of the UEFA champions' league for national championships; the case of Germany. Nyon: UEFA/IASE Seminar; May 2nd, 2006.

6. GOUGET JJ, PRIMAULT D. The economic impact of the UEFA Champions League on the French football championship. Nyon: UEFA/IASE Seminar; May 2nd, 2006.

7. JENNETT N. Attendances, Uncertainty of Outcome and Policy in Scottish Football League. Scot J Polit Econ. 1984; 33:176-198.

8. KONING RH. Balance in competition in Dutch soccer. The Statistician. 2000; 49(2):419431.

9. McMiLlAN, J. (1997). Rugby meets economics. New Zealand Economic Papers, 31(1), 93114.

10. MICHIE, J., OUGHTON C. Competitive Balance in Football: Trends and Effects. London: The Sports Nexus; 2004.

11. NEALE, W. C. (1964). The peculiar economics of professional sports. The Quarterly Journal of Economics, 78(1), 1.

12. NOVOTNY J., Sport economics $-1^{\text {st }}$ Ed. Prague: Wolters Kluwer CZ, 2011. ISBN 978-807357-666-0

13. QUIRK J, FORT R. Pay Dirt: The Business of Professional Team Sports. Princeton: Princeton University Press; 1992.

14. ROTTENBERG, S. (1956). The baseball players' labour market. The Journal of Political Economy, 64(3), 242-258. 
15. STAUDOHAR, P. D. (1999). Salary caps in professional team sports. Competition Policy in Professional Sports: Europe after the Bosman Case, 71-89.

16. SZYMANSKI S. The Economic Design of Sporting Contests. J Econ Lit. 2003; 41:11371187.

17. TENREIRO F. Can European football David's equal their Goliath's? The Portuguese case assessment. Nyon: UEFA/IASE Seminar; May 2nd, 2006

18. VROOMAN, J. (2000). The economics of American sports leagues. Scottish Journal of Political Economy, 47(4), 364-398.

Internet references:

1. Official web side of SANZAR and Super Rugby:

(http://www.sanzarrugby.com/superrugby/about-super-rugby/)

2. Official web side of Premiership Ruby: (www.premiershiprugby.com/history/ 